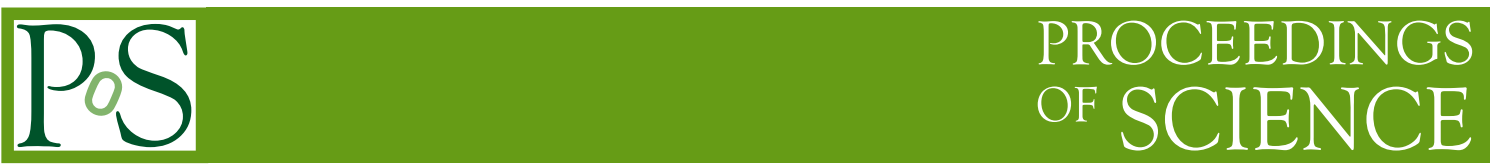

\title{
Muonless Events in ICAL at INO
}

\author{
Ali Ajmi ${ }^{a}$ and S. Uma Sankar ${ }^{b}$ \\ ${ }^{a}$ Homi Bhabha National Institute \\ Anushaktinagar, Mumbai 400 094, India. \\ ${ }^{b}$ Indian Institute of Technology Bombay \\ Mumbai 400 076, India. \\ E-mail: aliajmidtifr.res.in, uma@phy.iitb.ac.in
}

The primary physics signal events in the Iron Calorimeter at India-based Neutrino Observatory are the $v_{\mu}$ charged current (CC) interactions with a well defined muon track. Apart from these events, Iron Calorimeter can also detect other types of neutrino interactions, i.e. the electron neutrino charged current interactions and the neutral current events. It is possible to have a dataset containing mostly $v_{e} \mathrm{CC}$ events, by imposing appropriate selection cuts on the events. The $v_{\mu} \mathrm{CC}$ and the neutral current events form the background to these events. This study uses Monte Carlo generated neutrino events, to design the necessary selection cuts to obtain a $v_{e} \mathrm{CC}$ rich dataset. An optimized set of constraints are developed which balance the need for improving the purity of the sample and having a large enough event sample. Depending on the constraints used, one can obtain a neutrino data sample with the purity of $v_{e}$ events varying between $55 \%$ to $70 \%$.

The European Physical Society Conference on High Energy Physics

22-29 July 2015

Vienna, Austria 


\section{Introduction}

India-based Neutrino Observatory or the INO, an upcoming experimental facility will house the Iron Calorimeter (ICAL). The ICAL aims to study the interactions of atmospheric neutrinos and antineutrinos [1]. It is a giant magnetized neutrino detector, with Resistive Plate Chambers (RPCs) as the active detector elements [2, 3, 4]. It comprises of 3 modules, with $~ 30,000$ RPCs, and 151 iron layers weighing $\sim 50 \mathrm{kton}$ in total. A sketch of ICAL is shown in figure 1.

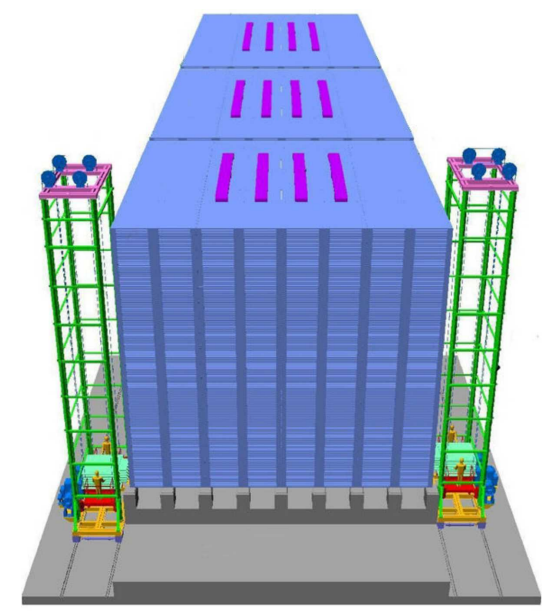

Figure 1: A Schematic Sketch of the INO Detector.

The RPC layers are interspaced with iron plates of $5.6 \mathrm{~cm}$ thickness. This enables the detector to obtain clearer muon tracks. Simultaneously, this also leads to the absorption of most electrons.

\section{The $v$-events at ICAL}

Charged current (CC) neutrino interactions produce leptons of corresponding neutrino flavor, while the neutral current (NC) interactions do not give any lepton. Muons produced in the $v_{\mu} \mathrm{CC}$ interactions form tracks in the detector. The $v_{e} \mathrm{CC}$ events give electrons which can produce electromagnetic showers, but no track can be seen. In case of the NC events, only the hadrons can be observed in the detector. The $v_{\mu}$ s with lower energy and in the horizontal direction [5] are confined to a few layers, with no clear muon track.

Events in ICAL at INO can be classified into events with muon tracks and those without such tracks. We refer the latter as the "muonless" events, which basically comprise the $v_{e} \mathrm{CC}$ events, "others" (all NC and few $v_{\tau} \mathrm{CC}$ ) and the low energy or horizontal $v_{\mu} \mathrm{CC}$ events.

The following study is done with neutrino interactions generated by the Nuance neutrino event generator [6] equivalent to 500 years kton data. The generated events are then simulated in the ICAL detector using GEANT4.

Applying certain selection cuts, we can obtain an events sample rich in atmospheric $v_{e} \mathrm{CC}$ events. The cuts are based mostly on the number of hits and number of layers, which are discussed in the following section. 


\section{Hits and layers}

A signal picked up by the copper strips of the RPC is referred to as a "hit". The detector thus records a number of hits during an event. We study the distributions of these hits for the $v_{\mu} \mathrm{CC}$ events, $v_{e} \mathrm{CC}$ events, and the $\mathrm{NC}$ events, in different ranges of $\mathrm{E}_{v}$, as shown in figure 2 and 3.
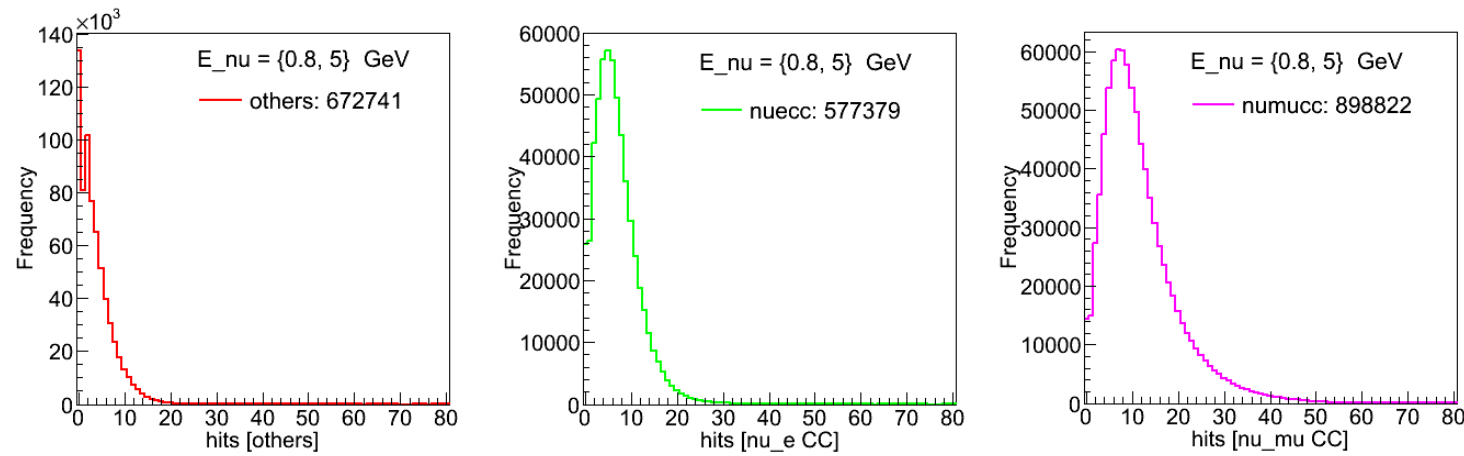

Figure 2: Hits Disribution in the neutrino energy bin $\mathrm{E}_{v}=\{0.8,5.0\} \mathrm{GeV}$.
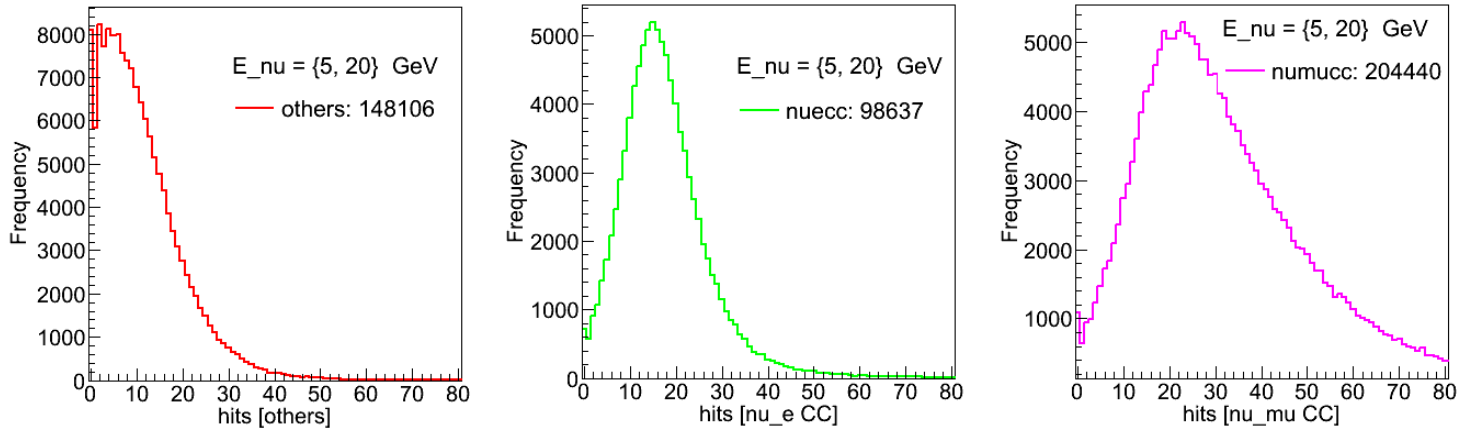

Figure 3: Hits Disribution in the neutrino energy bin $\mathrm{E}_{v}=\{5.0,20.0\} \mathrm{GeV}$.

The number of hits in $v_{\mu} \mathrm{CC}$ events is greatly enhanced with the increase in energy. The increment in number of hits (h) is much less for $v_{e} \mathrm{CC}$ events and is hardly seen in case of the NC events. So, a lower threshold of $\sim 10$ hits suppresses a large fraction of the $\mathrm{NC}$ events and the low energy $v_{e}, v_{\mu} \mathrm{CC}$ events.

The muons of the $v_{\mu} \mathrm{CC}$ events travel through several layers. So, an upper cut on the number of layers (L) removes most events with $\mu$-tracks.

\section{Selection of $v_{e} \mathrm{CC}$ rich events sample}

We aim to select an events sample of $v_{e} \mathrm{CC}$ events with minimum contamination from the $\mathrm{NC}$ or the $v_{\mu} \mathrm{CC}$ events. Various selection criteria have been devised [7] and a few of them are discussed here. 


\subsection{Average hits per layer}

The $e^{-} / e^{+} \mathrm{s}$ travel shorter distance than the hadrons. The muon tracks give mostly $2-3$ hits in a layer. So, a $v_{e} \mathrm{CC}$ event gives hits in a fewer layers than an event with a muon track, of equivalent energy. A lower cut on the average hits per layer (hpl) should eliminate events containing $\mu$ tracks.

\subsection{Maximum Hits Difference (mhd)}

The $v_{e} \mathrm{CC}$ events contain electrons, which lead to electromagnetic (EM) showers and hence should give a huge number of hits. Most of the electrons are absorbed by thick iron layers. However, if the shower starts at the edge of the iron layer, a sudden increment in the number of hits in the following layer is expected. This difference in the number of hits in the two adjacent layers in an event is calculated and is maximized over all such pairs in that event. A cut on this value of mhd selects more $v_{e} \mathrm{CC}$ events, as shown in table 1.

\begin{tabular}{|c|c|c|c|c|}
\hline Selection Criteria & $v_{e} \mathbf{C C}$ & others & $v_{\mu} \mathbf{C C}$ & $v_{e}$ CC purity \\
\hline $\mathrm{h}>10 ; \mathrm{L} \leq 5 ;$ & 163807 & 82717 & 107350 & $46 \%$ \\
\hline $\mathrm{h}>10 ; \mathrm{L} \leq 5 ; \mathrm{mhd}>5$ & 82500 & 34701 & 38824 & $53 \%$ \\
\hline $\mathrm{h}>15 ; \mathrm{L} \leq 5 ;$ & 68702 & 32953 & 36211 & $50 \%$ \\
\hline $\mathrm{h}>15 ; \mathrm{L} \leq 5 ; \mathrm{mhd}>5$ & 50295 & 21844 & 23991 & $52 \%$ \\
\hline
\end{tabular}

Table 1: Effect of the cut on the mhd (maximum hits difference). [500 years NH data in $\mathrm{E}_{v}=\{0.1,100\} \mathrm{GeV}$. $]$

\subsection{Comparing the hits in each layer}

The underlying principle of this criterion also rests on the concept of the EM shower. It attempts to recognise a pattern in the number of hits in adjacent layers, in two possible ways. We seek events with additional 5-6 hits in the next layer. Alternatively, we also look for events with a majority of the hits present in one layer. We define majority as $50 \%$ or $60 \%$ of the total number of hits. Effect of this selection criterion is shown in table 2.

\begin{tabular}{|c|c|c|c|c|}
\hline Selection Criteria & $v_{e} \mathbf{C C}$ & others & $v_{\mu} \mathbf{C C}$ & $v_{e}$ CC purity \\
\hline hits $>15 ;$ layers $\leq 5 ;$ & 68702 & 32953 & 36211 & $50 \%$ \\
\hline hits $>15 ;$ layers $\leq 5 ; \mathrm{h}_{L}>\mathrm{h}_{L \pm 1}+5$ & 47009 & 21191 & 22934 & $52 \%$ \\
\hline hits $>15 ;$ layers $\leq 5 ; \mathrm{h}_{L}>50 \%$ hits & 38479 & 13745 & 16934 & $56 \%$ \\
\hline hits $>15 ;$ layers $\leq 5 ; \mathrm{h}_{L}>60 \%$ hits & 29123 & 9038 & 11948 & $58 \%$ \\
\hline
\end{tabular}

Table 2: Event counts in the sample selected by the cut. [500 years $\mathrm{NH}$ data in $\mathrm{E}_{v}=\{0.1,100\} \mathrm{GeV}$.]

\subsection{Overall Hits Pattern (rms)}

The hits in different layers of $v_{e} \mathrm{CC}$ events are non-uniform. The hits are mostly over concentrated in some layers, while entirely sparse in the rest (owing to the EM shower nature). This is reflected in a layerwise hits distribution, in figure 4. 

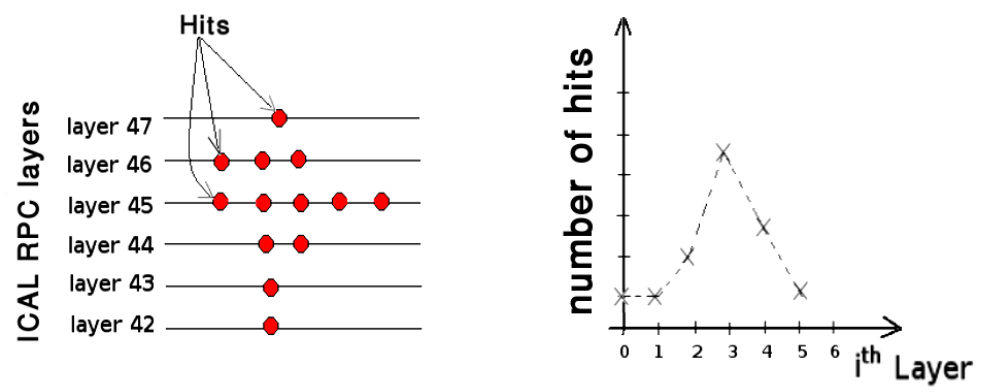

Figure 4: Schematic Diagram of hits in the RPC layers: the hit pattern among various layers in an event (left panel) and the number of hits vs layer number (right panel)

In the right panel of figure 4, the lowest layer hit is labelled to be $\mathbf{0}$, the next layer is $\mathbf{1}$ and so on. In such a plot, the $v_{\mu} \mathrm{CC}$ gives a broader peak than the $v_{e} \mathrm{CC} / \mathrm{NC}$. So, selecting events with such sharper peaks leads to rejecting a major fraction of $v_{\mu} \mathrm{CC}$ events. We parametrize this criteria by either the mean or RMS value of this distribution, and the effect is shown in table 3.

\begin{tabular}{|c|c|c|c|c|}
\hline Selection Criteria & $v_{e} \mathbf{C C}$ & others & $v_{\mu} \mathbf{C C}$ & $v_{e} \mathrm{CC}$ purity \\
\hline $\mathrm{h}>15 ; \mathrm{L} \leq 5$ & 68702 & 32953 & 36211 & $50 \%$ \\
\hline $\mathrm{h}>15 ; \mathrm{L} \leq 5 ; \mathrm{rms}<1.2$ & 56254 & 24916 & 25431 & $53 \%$ \\
\hline $\mathrm{h}>10 ; \mathrm{L} \leq 4$ & 125321 & 56177 & 62113 & $51 \%$ \\
\hline $\mathrm{h}>10 ; \mathrm{L} \leq 4 ; \mathrm{rms}<1.2$ & 111858 & 47961 & 52860 & $53 \%$ \\
\hline
\end{tabular}

Table 3: Effect of the rms cuts ( $r m s$ is the RMS or Root Mean Square of the layerwise hits distribution). [500 years $\mathrm{NH}$ data in $\mathrm{E}_{v}=\{0.1,100\} \mathrm{GeV}$.]

\section{Effects of Combined selection cuts}

Appropriate combination of these criteria improves the percentage of purity of $v_{e} \mathrm{CC}$ events in the selected sample. A few such combinations are shown in table 4.

\begin{tabular}{|c|c|c|c|c|}
\hline Selection Criteria & $v_{e} \mathbf{C C}$ & others & $v_{\mu} \mathbf{C C}$ & $v_{e} \mathrm{CC}$ purity \\
\hline $\mathrm{h}>10 ; \mathrm{L} \leq 4 ; \mathrm{rms}<1.2 ;$ max hits diff. $>3$ & 86157 & 35115 & 37026 & $54 \%$ \\
\hline $\mathrm{h}>10 ; \mathrm{L} \leq 5 ; \mathrm{rms}<1.2 ;$ max hits diff. $>3$ & 99814 & 43409 & 46455 & $56 \%$ \\
\hline $\mathrm{h}>10 ;$ mean $<2 ; \mathrm{rms}<1.2 ;$ max hits diff. $>3$ & 83954 & 35130 & 36127 & $54 \%$ \\
\hline $\mathrm{h}>10 ;$ mean $<2 ; \mathrm{rms}<1.2 ;$ max hits diff. $>5$ & 60959 & 23063 & 24129 & $56 \%$ \\
\hline $\mathrm{h}>10 ;$ mean $<2 ; \mathrm{rms}<1.2 ;$ max hits diff. $>5 ; \mathrm{hpl}>4$ & 51249 & 18247 & 18922 & $58 \%$ \\
\hline
\end{tabular}

Table 4: Effects of combined selection criteria. [500 years NH data in $\mathrm{E}_{v}=\{0.1,100\} \mathrm{GeV}$.]

\section{Results and Conclusion}

The hits and layers criteria can alone fetch a $v_{e} \mathrm{CC}$ sample of $\sim 50 \%$ purity. With the additional selection criteria, we can improve the purity of the sample. The most effective criteria are listed here along with the sample-sizes: 


\begin{tabular}{|c|c|c|}
\hline Selection Criteria & $v_{e}$ CC purity & Sample size (500 y) \\
\hline Maximum Hits diff. & $53 \%$ & 156,000 \\
\hline Overall Hits Pattern & $58 \%$ & 88,000 \\
\hline Comparing hits in layers & $60 \%$ & 43,000 \\
\hline Single layer hits & $68 \%$ & 6,500 \\
\hline
\end{tabular}

Table 5: Obtainable $v_{e}$ CC purity in the total sample and the corresponding sample size for 500 years of NH data.

The purity of $v_{e} \mathrm{CC}$ events in the total sample decreases with increasing sample size. It has also been observed that attempts to improve on the purity depletes the vertical events fraction. Appropriate selection cuts can be applied, while retaining optimum sample-sizes. This leads to a maximum $v_{e} \mathbf{C C}$ purity of $\sim \mathbf{6 0 \%}$ with $\sim 100$ events per year. We can also obtain an event sample with $v$ NC purity of $\sim 47 \%$ with $\sim 1800$ events per year, provided noise is under control [7].

The contribution of the muonless events in determining the neutrino mass hierarchy is not zero, rather $\sim 1$. But the statistical fluctuations in the data are too large for this contribution to have a significant effect [7].

\section{References}

[1] Anushree Ghosh, Tarak Thakore, and Sandhya Choubey. Determining the Neutrino Mass Hierarchy with INO, T2K, NOvA and Reactor Experiments. JHEP, 1304:009, 2013.

[doi:10.1007/JHEP04 (2013)009]

[2] V.M. Datar, Satyajit Jena, S.D. Kalmani, N.K. Mondal, P. Nagaraj, et al. Development of glass resistive plate chambers for INO experiment. Nucl.Instrum.Meth., A602:744-748, 2009.

[doi:10.1016/j.nima.2008.12.129]

[3] M. Sajjad Athar et al. India-based Neutrino Observatory: Project Report. Volume I., 2006. [url: http://www.ino.tifr.res.in/ino/OpenReports/INOReport.pdf]

[4] Naba K. Mondal. India-based Neutrino Observatory. Pramana, 79:1003-1020, 2012. [doi:10.1007/s12043-012-0444-9]

[5] Y. Fukuda et al. Study of the atmospheric neutrino flux in the multi-GeV energy range. Phys.Lett., B436:33-41, 1998. [doi:10.1016/S0370-2693(98)00876-4]

[6] D. Casper. The Nuance neutrino physics simulation, and the future. Nucl.Phys.Proc.Suppl., 112:161-170, 2002. [doi:10.1016/S0920-5632(02)01756-5]

[7] Ali Ajmi and S. Uma Sankar. Muonless Events in ICAL at INO. JINST, 10(04):P04006, 2015. [doi:10.1088/1748-0221/10/04/P04006, arXiv:1501.03252] 